\title{
Les déterminants de l'adoption de certaines bonnes pratiques culturales avant récolte de la noix de cajou en Côte d'Ivoire
}

\author{
Dr OUATTARA Gniré Mariam \\ Université Alassane OUATTARA de Bouaké-Côte d'Ivoire
}

\begin{abstract}
Résumé: L'objectif de cet article est d'identifier les déterminants de l'adoption des bonnes pratiques culturales de la noix de cajou. Les résultats de l'estimation du modèle logit multinomial non ordonné montrent que les variables sociales telles que la localisation et le niveau d'éducation (primaire, lycée et autre formation) favorisent l'adoption des bonnes pratiques de la noix de cajou avant la récolte. Par contre, l'adoption de certaines bonnes pratiques culturales au niveau de l'anacarde diminue avec l'âge $d u$ chef d'exploitant ainsi que la taille du ménage. À cet effet, les structures d'encadrement doivent cibler des formations complémentaires sur les techniques agricoles dans les régions de production afin d'améliorer les taux d'une adoption des bonnes pratiques culturales au niveau de la noix de cajou. Ce qui va induire une dynamique durable de croissance des rendements agricoles dont les avantages se feront ressentir à la fois sur les exploitants agricoles mais aussi au niveau macroéconomique.
\end{abstract}

Mots-clés : Bonnes pratiques culturales, noix de cajou ou anacarde, Côte d'Ivoire, multinomial.

Summary: This article purports to identify the determinants of the adoption of good agricultural practices of the cashew nut. The results of the estimation of the unordered multinomial logit model show that social variables such as location and level of education (primary, secondary and other) favor the adoption of good cashew practices prior to harvest. On the other hand, the adoption of certain cashew nut good farming practices decreases with the age of the farmer and the size of the household. To this end, supervisory structures should target further training on agricultural techniques in production regions in order to improve the rates of adoption of good cultivation practices at the cashew nut level. This will induce a sustained dynamic of growth in agricultural yields, the benefits of which will be felt not only on farmers but also at the macroeconomic level.

Keywords : Good agricultural practices, cashew nuts, Côte d'Ivoire, multinomial.

Jel :Q1;O3

\section{Introduction}

Introduit depuis l'indépendance, l'anacardier a d'abord été considéré comme un arbre de reboisement (1959- 1960) et ensuite comme un arbre de production fruitière (de 1960 à nos jours), (MINAGRI ${ }^{1}, 1999$ ). Aujourd'hui, le sous-secteur de l'anacarde en Côte d'Ivoire est devenu une filière agro-industrielle importante pour l'économie ivoirienne et une source de revenu appréciable pour les populations vivant en milieu rural. En effet, cette culture occupe plus de 250000 producteurs et fait vivre environ 2,5 millions de personnes.

En outre, la production nationale a connu un essor considérable, passant de 19000 tonnes en 1998 à plus de 700000 tonnes en 2015, hissant ainsi la Côte d'Ivoire au rang de premier producteur mondial de noix de cajou. La production ivoirienne représente près de $20 \%$ de l'offre mondiale de noix brutes de cajou. Malgré cette croissance rapide, la culture de l'anacardier est basée sur des techniques extensives (insuffisance de l'encadrement, non application de l'itinéraire technique, insuffisance du matériel végétal performant ...), entrainant un faible niveau de rendement $(500 \mathrm{~kg} / \mathrm{hectare}$ contre au moins 1000 à $1500 \mathrm{~kg} / \mathrm{hectare}$ en Inde et au Brésil).

Face à ce constat, et vu le poids socio-économique que suscite cette spéculation, l'État de Côte d'Ivoire a procédé à la réforme de la filière anacarde en 2013, afin de garantir la durabilité de sa culture. Pour rendre opérationnel cette réforme, six (06) axes stratégiques dont celui relatif à la formation et à l'encadrement de l'ensemble des producteurs ont été identifiés. La mise en œuvre de cet axe devrait se traduire par la vulgarisation d'un ensemble d'innovations et le suivi de l'application de ces bonnes pratiques agricoles par les producteurs. Pour ce faire, une convention tripartite a été signée entre le Conseil du Coton et de l'anacarde, le FIRCA et l'ANADER, suivie de la signature de contrats spécifiques entre le FIRCA et la Direction Générale de l'ANADER ainsi que ses cinq Directions Régionales, pour la mise en œuvre du conseil agricole dédié aux producteurs d'anacarde sur une période de 4 ans.

Après une année de mise en œuvre, 102282 producteurs dont $12 \%$ de femmes, issus 3110 villages, ont été formés aux bonnes pratiques agricoles par 150 conseillers agricoles (CAA) appuyés par 34 techniciens supérieurs (TSA) et 34 techniciens supérieurs en organisation professionnelle agricole (TSOPA). Pour la

${ }^{1}$ Ministère de l'Agriculture 
deuxième année, 149600 producteurs ont été formés par 220 CAA. Par ailleurs, depuis 2000, des partenaires techniques et financiers (GIZ/ iCA, GIZ/Profiab, RONGEAD, INADES Formation, CHIGATA) ont mis en œuvre des programmes de formation des producteurs, afin de leur permettre de maîtriser l'itinéraire technique de l'anacardier.

En dépit de ces efforts et d'une connaissance relativement acceptable des bonnes pratiques agricoles par les producteurs formés, force est de constater que l'application et/ou l'adoption de ces acquis restent encore problématiques. Les bonnes pratiques culturales peinent à être mises en œuvre par les producteurs. Pourtant, elles contribuent à générer des produits de qualité, à améliorer les revenus d'exportation ainsi que les conditions de vie des populations (FAO ; 2002). En ce qui concerne la littérature, plusieurs auteurs, tels que Henning et Cardona (2000), Khanna (2001), Kim et al. (2005), Mariano et al. (2012), Nyaupane et Gillespie (2009), Paudel et al. (2008), Ward et al. (2008), ont considéré les bonnes pratiques comme sources d'innovation et donc pouvant améliorer le rendement.

Aussi la théorie économique de l'innovation induite en agriculture met-elle en exergue le lien entre les choix technologiques des agriculteurs et les contraintes de leur environnement. Les facteurs affectant l'adoption de ces bonnes pratiques sont nombreuses (Kebede, Gunjal et al. 1990, Feder et Umali 1993, Wu et Babcock 1998, Foltz et Chang 2002, Barham, Foltz et al. 2004, Abdulai and Huffman 2005, Sauer et Zilberman 2009). Quels sont donc les déterminants de l'adoption des bonnes pratiques culturales de la noix de cajou dans le contexte ivoirien?

L'objectif de ce travail est d'identifier les déterminants de l'adoption de bonnes pratiques culturales avant récolte, de la noix de cajou concernant l'échantillon de l'étude. De façon spécifique, il s'agit d'abord de déterminer les facteurs socio-économiques pouvant influencer l'adoption de bonnes pratiques culturales avant la récolte de la noix de cajou en Côte d'Ivoire et ensuite de faire une comparaison par genre et par régions.

La seconde section consiste à une revue de littérature des déterminants des bonnes pratiques culturales et permet de mettre en évidence ceux qui semblent plus pertinents à analyser dans le cadre de la culture de la noix de cajou. La troisième section présente les méthodes utilisées pour déterminer les facteurs d'adoption des bonnes pratiques culturales de noix de cajou. Enfin, la quatrième section consiste à présenter les résultats et les interprétations ainsi que la conclusion et les implications de politiques qui en découlent.

\section{Revue de littérature}

De nos jours, dans la littérature économique, il est difficile de résumer l'effet des déterminants socioéconomiques et même agronomiques de l'adoption d'innovations agricoles et de bonnes pratiques culturales. Cette revue s'articule autour des travaux récents sur l'adoption d'innovations et de bonnes pratiques agricoles.

\subsection{Les facteurs endogènes}

Il s'agit des caractéristiques du producteur et de l'exploitation, notamment la taille, le degré de spécialisation, l'âge et la formation. Hall et al. (2009) indiquent qu'un système d'innovation est constitué d'individus et d'organisations demandant et fournissant des connaissances et des technologies, ainsi que des règles et mécanismes en matière de politiques qui influent sur la manière dont différents agents interagissent pour diffuser, obtenir et échanger des connaissances. De ce fait, le concept contemporain de l'innovation n'est pas seulement la production d'objets technologiques, c'est aussi l'amélioration en termes de performances au niveau institutionnel et politique pour la mise en pratique des connaissances (Smitts 2002).

Adéoti R.et al. (2002) utilisent un modèle économétrique Logit comme outil pour analyser l'utilisation des différentes technologies et déterminer les facteurs affectant leur adoption dans huit pays. Les résultats ont montré que la taille des ménages, le contact avec les services de vulgarisation, les revenus extra-agricoles et le niveau d'éducation affectent positivement la probabilité d'adopter les nouvelles variétés et les extraits de neem et papayer. Par contre, la superficie des champs de niébé affecte négativement l'utilisation des extraits de feuilles de neem.

Ichaou Mounirou (2015) a identifié les déterminants de la perception de l'adoption des innovations techniques agricoles dans le bassin cotonnier de Banikoara au Bénin. Les résultats de l'estimation du modèle logit multinomial montrent que les variables telles que l'âge, le niveau d'éducation et d'instruction et les risques et incertitudes ne favorisent pas une bonne perception de l'adoption des innovations techniques agricoles dans la culture du coton et des productions vivrières (maïs, manioc, arachide et mil).

Lawal et al. (2010) ont trouvé également que l'accès aux crédits est un facteur très déterminant de la production d'anacarde et donc du revenu obtenu de la vente de ses produits. Par rapport au contexte de Madagascar, Randrianarisoa et Minten (2003) insistent, dans l'étude de l'adoption des engrais, sur l'adéquation de l'innovation au milieu de vie des producteurs, en termes d'interdépendance des différents facteurs de production, notamment une bonne maîtrise de l'eau doublée d'une bonne connaissance de l'innovation de la part des agriculteurs. 
Savadogo et al. (1998) montrent que les revenus non agricoles et la taille du ménage agricole ont un impact positif sur la probabilité d'adoption de la traction animale dans les zones de climats guinéen et soudanosahélien du Burkina-Faso. Ils concluent que l'utilisation de la traction animale améliore la productivité marginale du facteur travail et les rendements agricoles, et que le recours simultané à la traction animale et aux engrais induisent de meilleurs résultats.

\subsection{Les facteurs exogènes}

Les facteurs exogènes sont constitués de deux facteurs, notamment ceux non maitrisables (réglementation, conditions climatiques) et des facteurs partiellement maitrisables comme l'accès à l'information ainsi que le niveau de sensibilisation à l'innovation.

Khanna (2001) met en évidence l'existence d'un effet de la zone de production sur l'adoption de pratiques de fertilisation parcellaire. Dans les zones de grandes cultures, les conditions pédoclimatiques peuvent contraindre les agriculteurs dans leurs choix de production. Des conditions climatiques comme des sécheresses répétées poussent les agriculteurs à rejeter certaines innovations pour des raisons techniques (Mariano, Villano et al. 2012).

Les études de Bougherara et al (2010) ainsi que celles de Giannocaro et Berbel (2013) montrent que les exploitants n'envisagent pas de changements radicaux dans l'utilisation des terres ou des intrants avec les évolutions futures de la Politique Agricole Commune. Au regard de ces différentes études, il ressort qu'il existe peu d'étude sur des déterminants des bonnes pratiques culturales dans le cas de la Côte d'Ivoire.

\subsection{Adoption d'innovation, bonne pratique culturale et rendement}

Balogoun (2010), utilisant les données relatives aux caractéristiques socio-économiques des producteurs d'anacarde, aux pratiques de gestion des plantations, aux intrants et aux revenus de la production collectée dans les principales zones de production du Bénin, montre que le manque d'entretien des plantations et d'intrants ne permettent pas une augmentation substantielle des rendements en pomme et en noix au niveau des différentes zones de production. Mais le revenu annuel obtenu de la vente des noix d'anacarde est significativement influencé par la superficie plantée, le coût total de la main-d'œuvre, le rendement des noix, le prix de vente du kilogramme de noix, la situation matrimoniale du producteur et l'accès aux crédits.

Kouakou (2014) traite de la mesure de la performance de la culture du coton en Côte d'Ivoire. Il identifie les déterminants de l'efficacité ou de l'inefficacité des agriculteurs. À partir d'une frontière stochastique de production et d'une frontière de coût, il estime les niveaux d'efficacité technique, d'efficacité allocative et d'efficacité économique. Il ressort qu'aucune zone de production n'a le monopole de performances. L'inefficience constatée s'explique par le faible niveau d'encadrement et les fluctuations des prix bords-champs. L'amélioration de l'efficacité passe par une intensification du niveau d'encadrement technique des paysans et la mise en place d'une politique attrayante de prix du coton.

\section{Approche méthodologique}

Cette partie de l'étude s'articule autour de deux objectifs. Il s'agit de la modélisation de la décision d'adoption de bonne pratique culturale, afin de déterminer les facteurs qui influent sur la décision d'un agent face au choix de l'adoption ou non de la bonne pratique culturale. Le cadre s'articule autour de deux points, à savoir le modèle théorique et le modèle empirique.

\subsection{Modèle d'adoption des bonnes pratiques culturales}

La plupart des études antérieures d'adoption utilisent un modèle multinomial inspiré de Cimmyt (1993). En effet, en supposant que chaque individu i ait à choisir entre quatre alternatives $(j=0$ à 4$)$ concernant la variable de bonne pratique avant la récole. Ce que nous cherchons à étudier est la décision unique d'un individu parmi un certain nombre d'alternatives non ordonnées. Dans un modèle de choix non ordonnés, l'individu i va comparer les différents niveaux d'utilité associés aux divers choix, puis opter pour celui qui maximise son utilité Uij parmi les J choix. Pour l'individu i, l'utilité du choix j.

La probabilité que l'individu $\mathrm{i}$, compte tenu de ses caractéristiques $X_{i k}$, fasse partie de la catégorie $\mathrm{j}$ est supposée dépendre des $X_{i k}$ ou, plus précisément, d'une combinaison linéaire des $X_{i k}$. Formellement, cela s'écrit : $P\left(j / X_{i}=\beta_{0 j}+\beta_{1 j} X_{i 1}+\beta_{2 j} X_{i 2}+\ldots \ldots+\beta_{i k}\right)=G\left(X_{i} \beta_{j}\right)$

Pour $\mathrm{j}=1,2, \ldots 4$, le vecteur $X\left(X_{i 1} ; X_{i 2} ; \ldots . . X_{i k}\right)$ est le vecteur (ligne) des variables explicatives du modèle. Sa première composante vaut systématiquement 1 . Elle prend en compte, dans le modèle, le fait que les 
catégories n'ont pas les mêmes effectifs. On remarquera que les paramètres de la combinaison linéaire dépendent de la catégorie j. On note $\beta_{j}\left(\beta_{0 j} ; \beta_{1 j} ; \beta_{2 j} ; \ldots . . \beta_{i k}\right)$.

Le problème est de trouver une forme fonctionnelle $\mathrm{G}$ telle que chaque quantité ( ) i $\mathrm{P} \mathrm{j}$ x soit bien une probabilité, c'est-à-dire possède les deux propriétés suivantes :

$0\left\langle P\left(j / X_{i}\right)<1 \mathrm{Et} \sum_{j=1}^{4}\left(j / X_{i}\right)=1(1)\right.$

Pour assurer la stricte positivité de $P\left(j / X_{i}\right)$, on prend la fonction exponentielle $P\left(j / X_{i}\right)=\exp \left(X_{i} \beta_{j}\right)$.

Mais cette quantité peut prendre des valeurs supérieures à 1.On la norme alors par la somme des $\exp \left(X_{i} \beta_{j}\right)$ et le modèle s'écrit : $P\left(j / X_{i}\right)=\frac{\exp \left(X_{i} \beta_{j}\right)}{\sum_{h=1}^{4} \exp \left(X_{i} \beta_{j}\right)}$

La forme fonctionnelle donnée répond bien aux deux propriétés. Le modèle possède a priori un nombre relativement élevé de paramètres : $(\mathrm{k}+1) \mathrm{x}$. Les effets des caractéristiques $\mathrm{X}$ sur l'appartenance à l'une des $\mathrm{j}$ catégories ne sont pas identifiés (les paramètres ne le sont pas).

Pour ce type de modèle, une infinité de valeurs de $\beta_{j}$ sont donc possibles, qui conduisent à une même valeur de la probabilité. Il faut alors imposer aux paramètres une condition qui permet l'identification du modèle. Celle qui est retenue en règle très générale est d'imposer la nullité de tous les paramètres relatifs à une catégorie donnée, appelée alors catégorie de référence.

Avec cette condition identifiante, le modèle s'écrit finalement :

$$
\left\{\begin{array}{l}
P\left(j^{\prime} X_{i}\right)=\frac{\exp \left(X_{i} \beta_{j}\right)}{1+\sum_{h=1}^{J-1} \exp \left(X_{i} \beta_{h}\right)} \text { pour } \mathrm{j}=1,2, \ldots . . \mathrm{J}-4 \\
P\left(j^{\prime} / X_{i}\right)=\frac{1}{1+\sum_{h=1}^{J-1} \exp \left(X_{i} \beta_{h}\right)}
\end{array}\right.
$$

\subsection{Les données et variables explicatives du modèle}

\subsubsection{Définition des variables utilisées}

En se focalisant sur la littérature, les variables socio-économiques et techniques ainsi que les hypothèses à tester sont contenues dans le tableau ci-dessous.

\begin{tabular}{|l|l|l|}
\hline Variables & Définitions & Hypothèses \\
\hline $\begin{array}{l}\text { Niveau } \\
\text { d'éducation }\end{array}$ & $\begin{array}{l}\text { Niveau d'éducation qualitative multinomiale } \\
\text { ordonnée du producteur d'anacarde }\end{array}$ & $\begin{array}{l}\text { Le niveau d'instruction pourrait influencer } \\
\text { positivement ou négativement l'adoption de la } \\
\text { technologie }\end{array}$ \\
\hline Sexe & $\begin{array}{l}\text { Variable binaire qui prend la valeur 1 si l'individu est } \\
\text { homme et 0 s'il est femme }\end{array}$ & $\begin{array}{l}\text { Le faite d'être une femme influence } \\
\text { négativement la probabilité d'adopter une } \\
\text { nouvelle technologie }\end{array}$ \\
\hline Formation & $\begin{array}{l}\text { La formation des producteurs ou encadrement, variable } \\
\text { binaire qui prend la valeur si le producteur à bénéficier de } \\
\text { formation ou d'encadrement et 0 si non. }\end{array}$ & $\begin{array}{l}\text { La formation des producteurs aux bonnes } \\
\text { pratiques culturales est positivement liée à leur } \\
\text { adoption }\end{array}$ \\
\hline $\begin{array}{l}\text { Localisation } \\
\text { Taille du ménage } \\
\text { Gontougo et 0 si en dehors de Gontougo }\end{array}$ & $\begin{array}{l}\text { Variable quantitative } \\
\text { positivement ou négativement l'adoption de } \\
\text { bonne pratique }\end{array}$ \\
\hline $\begin{array}{l}\text { Agge du chéside à } \\
\text { d'exploitation }\end{array}$ & Variable quantitative & $\begin{array}{l}\text { Le niveau d'instruction pourrait influencer } \\
\text { positivement ou négativement l'adoption de } \\
\text { bonne pratique }\end{array}$ \\
\hline $\begin{array}{l}\text { Nombre de } \\
\text { plantation }\end{array}$ & Variable quantitative & $\begin{array}{l}\text { L'âge pourrait influencer positivement ou } \\
\text { négativement l'adoption de bonne pratique }\end{array}$ \\
\hline
\end{tabular}




\subsubsection{Source des données}

Les données issues de cette étude proviennent d'une enquête réalisée en 2015 par le Conseil du Coton et de l'Anacarde (CCA), en collaboration avec l'initiative du cajou africain (iCA devenue ComCashew), sur la productivité des vergers d'anacarde en Côte d'Ivoire. Cette enquête avait pour objectif d'apprécier la rentabilité socio-économique des plantations d'anacarde. À terme, les résultats devraient permettre de mieux orienter les interventions et les soutiens aux producteurs de cajou dans leurs communautés. La population est estimée à 330 000 producteurs. L'échantillon retenu dans cette étude est de 503 producteurs et la répartition s'est faite par strate, soit 19 régions de production. Le critère de répartition était le niveau de production régionale annuelle.

\subsection{Méthode de construction de l'indice de bonnes pratiques culturales}

La démarche de construction de l'indice découle des indicateurs utilisés pour approcher les différentes pratiques culturales avant la récolte. En effet, dans le questionnaire, les différentes pratiques culturales d'avant la récolte sont appréhendées à travers des questions dichotomiques indépendantes. Cinq (5) questions dichotomiques sont identifiées à cet effet. De ce fait, le nombre total de bonnes pratiques culturales possibles est de 55. Ainsi, la méthode adéquate est de détecter les bonnes pratiques importantes qui illustrent au mieux le comportement des producteurs. La démarche itérative a été utilisée. En effet, chaque étape de cette démarche consiste à construire un axe factoriel à partir de l'Analyse Factorielle des Correspondances (AFC). Ensuite, on retient comme critères importants ceux dont l'inertie est supérieure à la valeur moyenne des inerties (règle de Kaiser). Après cette étape, on obtient un ensemble de 50 possibilités. On reprend le processus jusqu'à ce que le nombre de possibilités à la dernière étape vaille 2 .

\subsubsection{Résultat de construction de l'indice des bonnes pratiques culturales avant récolte}

Dans le cadre de cette recherche, le processus s'est arrêté à la deuxième étape. À la première étape, les pratiques culturales d'avant récolte retenues sont : tailler les arbres, désherber la plantation et la protéger contre les insectes. La pratique consistant à éviter les feux de brousse est jugée non importante pour la construction, tel que le tableau de l'annexe 1 le montre.

À la deuxième étape, on retrouve comme bonnes pratiques culturales d'avant récolte, tailler les arbres, désherber et protéger la plantation contre les insectes. On déduit que les bonnes pratiques culturales d'avant récolte pratiqués par les producteurs peuvent être formées à partir des 3 pratiques suivantes : tailler les arbres, protéger la plantation contre les insectes et désherber. (Annexe 2).

Parmi les bonnes pratiques culturales d'avant récolte, le désherbage (simple) et l'élagage associé au désherbage, sont privilégiées par les producteurs. Ils sont pratiqués respectivement à 15,45\% et à $70 \%$ par les producteurs. La lutte contre les insectes ravageurs est peu pratiquée, cela pourrait se traduire par le fait que les méthodes de lutte contre les ravageurs ne sont pas bien connues des producteurs.

Tableau 1: Pratiques culturales avant la récolte

\begin{tabular}{|l|l|l|}
\hline Bonnes pratiques culturales & $\mathbf{N}$ & $\mathbf{\%}$ \\
\hline Aucune & 2 & 0,44 \\
\hline Eviter la présence d'insectes ravageurs & 12 & 2,65 \\
\hline Enlever les branches mortes et malades & 10 & 2,21 \\
\hline Désherbage/coupure des mauvaises herbes & 70 & 15,45 \\
\hline Enlever les branches mortes et désherber les mauvaises herbes & 359 & 79,25 \\
\hline Total & 453 & 100 \\
\hline
\end{tabular}

Source : L'auteur à partir des données l'Enquête CCA-iCA 2015

\subsection{Analyse et discussion des résultats économétriques}

Le tableau ci-dessous indique les facteurs explicatifs de l'adoption de bonnes pratiques culturales avant la récolte.

Tableau 2:Résultat de l'estimation du modèle logit multinomiale d'adoption des bonnes pratiques culturales de noix de cajou avant la récolte.

\begin{tabular}{|c|c|c|c|c|c|}
\hline Pratiques culturales avant la récolte & Variables & Coefficients. & Ecart type. & $\mathrm{Z}$ & $\mathrm{P}>\mathrm{Z}$ \\
\hline \multirow[t]{7}{*}{ Aucune } & Sexe & 2,032 & 1,517 & 1,340 & 0,181 \\
\hline & Age & $-0,450 * *$ & 0,223 & $-2,020$ & 0,044 \\
\hline & age 2 & $0,004 * * *$ & 0,002 & 1,690 & $\mathbf{0 , 0 9 0}$ \\
\hline & Résider à Gontougo & $6,345 *$ & 1,925 & 3,300 & $\mathbf{0 , 0 0 1}$ \\
\hline & \multicolumn{5}{|c|}{ Niveau d'éducation - Modalité de référence : aucun niveau } \\
\hline & Lycée & $2,827 * * *$ & 1,705 & 1,660 & 0,097 \\
\hline & Technique & $-12,729$ & 10601,260 & 0,000 & 0,999 \\
\hline
\end{tabular}


Déterminants de l'adoption de certaines bonnes pratiques culturales avant la récolte de la noix

\begin{tabular}{|c|c|c|c|c|c|}
\hline & Autre & $-15,405$ & 3415,611 & 0,000 & 0,996 \\
\hline & Nombres ménages & 0,090 & 0,058 & 1,550 & 0,122 \\
\hline & Nombres plantations & $-0,442$ & 1,452 & $-0,300$ & 0,761 \\
\hline & cons & 3,557 & 5,426 & 0,660 & 0,512 \\
\hline \multirow[t]{14}{*}{ Désherber } & Sexe & $1,417 * *$ & 0,628 & 2,260 & 0,024 \\
\hline & Age & $-0,101$ & 0,109 & $-0,920$ & 0,357 \\
\hline & Age 2 & 0,001 & 0,001 & 1,070 & 0,284 \\
\hline & \multicolumn{5}{|c|}{ Région - Modalité de référence: en dehors de Gontougo } \\
\hline & Résider à Gontougo & $-16,056$ & 5568,052 & 0,000 & 0,998 \\
\hline & \multicolumn{5}{|c|}{ Niveau d'éducation - Modalité de référence : aucun niveau } \\
\hline & Primaire & 1,067 & 0,664 & 1,610 & 0,108 \\
\hline & Collège & 1,296 & 0,809 & 1,600 & 0,109 \\
\hline & Lycée & $-14,035$ & 1945,246 & $-0,010$ & 0,994 \\
\hline & Technique & $-14,117$ & 6018,305 & 0,000 & 0,998 \\
\hline & Autre & $-12,232$ & 2423,924 & $-0,010$ & 0,996 \\
\hline & Nombres ménages & $-0,066$ & 0,052 & $-1,280$ & 0,200 \\
\hline & Nombres plantations & $-0,257$ & 0,479 & $-0,540$ & 0,592 \\
\hline & cons & $-1,775$ & 2,974 & $-0,600$ & 0,551 \\
\hline \multirow{14}{*}{$\begin{array}{l}\text { Tailler les arbres et protéger contre } \\
\text { les insectes }\end{array}$} & Sexe & $1,043 *$ & 0,393 & 2,650 & 0,008 \\
\hline & Age & $-0,024$ & 0,073 & $-0,320$ & 0,746 \\
\hline & age2 & 0,000 & 0,001 & 0,430 & 0,665 \\
\hline & \multicolumn{5}{|c|}{ Région - Modalité de référence : en dehors de Gontougo } \\
\hline & Résider à Gontougo & 0,691 & 0,903 & 0,770 & 0,444 \\
\hline & \multicolumn{5}{|c|}{ Niveau d'éducation - Modalité de référence : Aucun niveau } \\
\hline & Primaire & 0,528 & 0,374 & 1,410 & 0,158 \\
\hline & Collège & $-0,220$ & 0,670 & $-0,330$ & 0,743 \\
\hline & Lycée & $-0,465$ & 1,082 & $-0,430$ & 0,668 \\
\hline & Technique & $-14,368$ & 3198,285 & 0,000 & 0,996 \\
\hline & Autre & $2,500 *$ & 0,840 & 2,980 & $\mathbf{0 , 0 0 3}$ \\
\hline & Nombres ménages & $-0,030$ & 0,022 & $-1,350$ & 0,177 \\
\hline & Nombres plantations & $-0,127$ & 0,227 & $-0,560$ & 0,576 \\
\hline & _cons & $-2,045$ & 1,956 & $-1,050$ & 0,296 \\
\hline
\end{tabular}

Source : L'auteur, à partir des données l'Enquête CCA-iCA 2015.

Les résultats de cette étude révèlent les commentaires suivants. En effet, être un producteur résidant dans la région de Gontougo augmente la probabilité de pratiquer d'autres bonnes pratiques culturales qui sont différentes de celles énumérées dans l'étude, contrairement à un autre résidant hors de Gontougo. Ce résultat indiquerait que ces derniers comprennent le bien fondé des bonnes pratiques culturales agricoles.

La perception de l'adoption des bonnes pratiques agricoles est une fonction décroissante de l'âge. Les plus jeunes producteurs ont une prédisposition à l'acceptation des innovations par rapport aux producteurs plus âgés et au moins instruits quasi-définitivement acquis aux pratiques endogènes. En effet, la plupart des producteurs de la noix de cajou de l'étude ont un âge compris entre 19 et 91 ans. La moyenne d'âge dans les régions d'étude en Côte d'Ivoire est de 50 ans. Cette situation pourrait s'expliquer par l'âge relativement avancé des producteurs de la culture d'anacardier. Nos résultats vont dans le même sens que ceux de BALOGOUN (2010). Aussi, la majorité des terres appartiennent encore à des personnes âgées.

En ce qui concerne le niveau d'éducation, les résultats appellent les commentaires suivants. En effet, avoir un niveau lycée influence positivement la probabilité de développer d'autres bonnes pratiques en plus de celles citées dans le cadre de l'étude, contrairement à celui qui n'a aucun niveau de formation. Les producteurs, dont le niveau d'étude est supérieur, sont plus conscients des impacts tangibles, positifs et récurrents des bonnes pratiques agricoles. De même, le fait d'avoir reçu d'autres formations augmente la probabilité de tailler les arbres et de les protéger contre les insectes, comparativement à celui qui n'a aucun niveau d'étude.

En outre, le fait d'être un homme influence positivement la probabilité de désherber non seulement sa plantation mais aussi de tailler les arbres et de les protéger contre les insectes. Cette situation signifierait que les hommes ont plus de moyens physiques et financiers pour entretenir leurs champs.

\section{Conclusion}

La culture de l'anacardier constitue une activité économique très importante pour les planteurs des zones de production étudiées. Dans le cadre de l'analyse empirique, un modèle logit multinomial à modalités non ordonnées a été utilisé sur un échantillon d'exploitants agricoles spécialisés dans la culture de la noix de cajou dans les dix-neuf régions de production que compte la Côte d'Ivoire.

Les bonnes pratiques importantes qui illustrent au mieux le comportement des producteurs se situent à deux niveaux. Il s'agit, d'une part, des bonnes pratiques avant la récolte telles que désherber, tailler les arbres et les protéger contre les insectes ainsi que d'autres formes de pratiques et, d'autres part, des bonnes pratiques après la récolte. 
Le choix d'adopter une bonne pratique culturale avant la récolte est influencée par les variables sociales telles que la région de résidence du planteur, l'âge du chef d'exploitation, son niveau d'éducation et son sexe. Parmi ces variables, seul l'âge agit négativement sur certaines bonnes pratiques culturales. Les producteurs localisés dans la région de Gontougo sont plus enclins à enlever les branches mortes et malades de leur champ, contrairement à ceux qui ne disposent d'aucun niveau d'éducation et localisés en dehors de Gontougo.

En ce qui concerne les bonnes pratiques culturales, l'analyse économétrique montre que les planteurs adoptent six pratiques identifiées dans le cadre de l'étude. Celles-ci sont influencées par les variables sociales, mais aussi par une variable économique, à savoir la taille du ménage. En effet, le vieillissement des exploitants et la faiblesse de leur niveau d'instruction pourraient hypothéquer tout élan d'adoption de bonnes pratiques culturales avant la récolte.

Aussi, L'État doit mettre en œuvre des programmes spécifiques d'intensification de la formation technique des exploitants agricoles sur les bonnes pratiques culturales et contribuer au regroupement de ces derniers dans le cadre des coopératives. Ces deux facteurs seraient de nature à induire des taux élevés d'une bonne perception de l'adoption des bonnes pratiques de la culture de l'anacardier.

\section{Références bibliographiques}

[1]. Adégbola P. Y., Oloukoï L. et H. C. Sossou. 2005. Analyse de la compétitivité de la filière anacarde au Bénin. Rapport technique final, PAPA/INRAB, Bénin. 35 p.

[2]. Bougherara, D., Latruffe, L. (2010). Potential impact of the EU 2003 CAP reform on land idling decisions of French landowners: Results from a survey of intentions. Land Use Policy, 27(4): 1153-1159.

[3]. Dwomoh E. A., Ackonor J. B. and J. V. K. Afun. 2008. Survey of insect species associated with cashew (Anacardium occidentale Linn.) and their distribution in Ghana. Afr. J. Agric. Res. 3: 205 - 214

[4]. FAO (2002), rapport Bonnes pratiques agricoles1.

[5]. Feder G. \& Umali D.L., 1993, the adoption of agricultural innovations, Review. Technological forecasting and Social Change, 43, 215-239.

[6]. Giannoccaro, G., Berbel, J. (2013). Farmers' stated preference analysis towards resources use under alternative policy scenarios. Land Use Policy, 31: 145-155.

[7]. Gafsi M. \& Mbétid-Bessane.E., 2003, Stratégies des exploitations cotonnières et libéralisation de la filière. Cahiers Agricultures, $12,4,253-260$.

[8]. Greene W., 2005, Econométrie. Pearson Education, 5e éd., Paris, 943 p.

[9]. Ichaou Mounirou (2015) Perception et adoption des innovations techniques agricoles dans le bassin cotonnier de Banikoara au Bénin

[10]. Lawal J. O. and C.O. Jaiyeola. 2007. Economic analysis of cocoa wine produced from cocoa powder. J. Agri. Food and Environ. 5 (2): $76-77$.

[11]. Lawal J. O., Oduwole O. O, Shittu T. R. and A. A. Muyiwa. 2010. Profitability of value addition to cashew farming households in Nigeria. Afr. Crop Sci. J. 19 (1) : 49 - 54.

[12]. Mbétid-Bessane E., 2002, Gestion des exploitations agricoles dans le processus de libéralisation de la filière cotonnière en Centrafrique. Thèse de doctorat (unique) en Economie, Institut National Polytechnique, Toulouse, 317 p.

[13]. Mbétid-Bessane E., 2003, Crise cotonnière en Centrafrique et choix des agriculteurs en fonction de leur localisation à la ville. Tropicultura,

[14]. Nkamleu, G. B. et Coulibaly, O. (2000), « Les déterminants du choix des méthodes de lutte contre les pestes dans les plantations de cacao et café du sud-Cameroun ». Revue Economie Rurale No 259 Sept-Oct, 2000 pp 75-85.

[15]. Schmidt P \& Strauss RP, 1975. The prediction of occupation using multiple logit models. International Economic Review 16(2): 471-86.

[16]. Shapiro BI, Brorsen BW \& Doster DH, 1992. Adoption of double-cropping soybeans and wheat.

[17]. Southern Journal of Agricultural Economics 24: 33-40.

[18]. Smitts R, 2002. Etudes sur l'innovation au 21ème siècle: les questions des utilisateurs [Studies on innovation in the 21st century: User issues]. Prospective technologique et changement social 69:861-83.

[19]. Strauss D, 1979. Some results on random utility model. Journal of Mathematical Psychology 20(1):

[20]. 35-52.

[21]. Sumberg J, 2005. Systems of innovation theory and the changing architecture of agricultural research in Africa. Food Policy 30(1): $21-41$.

[22]. Hammed L. A., Amnikwe J. C. and A. R. Adededji. 2008. Cashew nuts and production development in

[23]. Mole P. N. 2000. Smallholder cashew development opportunities and linkages to food security in Nampula Province, Mozambique: Summary of findings and implications for.

Annexe

\begin{tabular}{|c|c|c|c|c|c|}
\hline Plantation & \multicolumn{2}{|l|}{ Catégories } & Poids & Qualité & $\begin{array}{l}\% \\
\text { inertie }\end{array}$ \\
\hline \multirow{8}{*}{ A } & \multirow{2}{*}{ Tailler les arbres } & Oui & $\mathbf{0 , 0 5 1}$ & 0,63 & 0,011 \\
\hline & & Non & 0,011 & 0,63 & 0,048 \\
\hline & \multirow{2}{*}{ Désherber la plantation } & Oui & $\mathbf{0 , 0 5 7}$ & 0,959 & 0,005 \\
\hline & & Non & 0,006 & 0,959 & 0,053 \\
\hline & \multirow{2}{*}{ Protéger la plantation contre les insectes } & Oui & $\mathbf{0 , 0 5 1}$ & 0,666 & 0,011 \\
\hline & & Non & 0,011 & 0,666 & 0,048 \\
\hline & \multirow{2}{*}{ Éviter les feux de brousse } & Oui & 0,017 & 0,466 & 0,043 \\
\hline & & Non & 0,045 & 0,466 & 0,016 \\
\hline \multirow{2}{*}{ B } & \multirow{2}{*}{ Tailler les arbres } & Oui & $\mathbf{0 , 0 5 1}$ & 0,63 & 0,011 \\
\hline & & Non & 0,011 & 0,63 & 0,048 \\
\hline
\end{tabular}


Déterminants de l'adoption de certaines bonnes pratiques culturales avant la récolte de la noix

\begin{tabular}{|c|c|c|c|c|c|}
\hline & \multirow{2}{*}{ Désherber la plantation } & Oui & $\mathbf{0 , 0 5 7}$ & 0,959 & 0,005 \\
\hline & & Non & 0,006 & 0,959 & 0,053 \\
\hline & \multirow{2}{*}{ Protéger la plantation contre les insectes } & Oui & 0,051 & 0,666 & 0,011 \\
\hline & & Non & 0,011 & 0,666 & 0,048 \\
\hline \multirow{8}{*}{$\mathrm{C}$} & \multirow{2}{*}{ Tailler les arbres } & Oui & $\mathbf{0 , 0 5 1}$ & 0,63 & 0,011 \\
\hline & & Non & 0,011 & 0,63 & 0,048 \\
\hline & \multirow{2}{*}{ Désherber la plantation } & Oui & $\mathbf{0 , 0 5 7}$ & 0,959 & 0,005 \\
\hline & & Non & 0,006 & 0,959 & 0,053 \\
\hline & \multirow{2}{*}{ Protéger la plantation contre les insectes } & Oui & 0,051 & 0,666 & 0,011 \\
\hline & & Non & 0,011 & 0,666 & 0,048 \\
\hline & \multirow{2}{*}{ Éviter les feux de brousse } & Oui & 0,023 & 0,42 & 0,037 \\
\hline & & Non & 0,04 & 0,42 & 0,021 \\
\hline \multirow{8}{*}{$\mathrm{D}$} & \multirow{2}{*}{ Tailler les arbres } & Oui & 0,045 & 0,624 & 0,016 \\
\hline & & Non & 0,017 & 0,624 & 0,043 \\
\hline & \multirow{2}{*}{ Désherber la plantation } & Oui & $\mathbf{0 , 0 5 7}$ & 0,959 & 0,005 \\
\hline & & Non & 0,006 & 0,959 & 0,053 \\
\hline & \multirow{2}{*}{ Protéger la plantation contre les insectes } & Oui & $\mathbf{0 , 0 5 1}$ & 0,666 & 0,011 \\
\hline & & Non & 0,011 & 0,666 & 0,048 \\
\hline & \multirow{2}{*}{ Éviter les feux de brousse } & Oui & 0,017 & 0,232 & 0,043 \\
\hline & & Non & 0,045 & 0,232 & 0,016 \\
\hline \multicolumn{3}{|c|}{ Moyenne } & 0,031 & & 0,0157 \\
\hline
\end{tabular}

Source : l'auteur à partir de l'enquête CCA-iCA 2015

\begin{tabular}{|c|c|c|c|c|c|}
\hline Plantation & \multicolumn{2}{|l|}{ Catégories } & Poids & Qualité & $\%$ inertie \\
\hline \multirow[t]{6}{*}{$\mathbf{A}$} & \multirow[t]{2}{*}{ Tailler les arbres } & Oui & 0,068 & 0,678 & 0,015 \\
\hline & & Non & 0,015 & 0,678 & 0,068 \\
\hline & \multirow[t]{2}{*}{ Désherber la plantation } & Oui & 0,076 & 0,956 & 0,008 \\
\hline & & Non & 0,008 & 0,956 & 0,076 \\
\hline & \multirow[t]{2}{*}{ Protéger la plantation contre les insectes } & Oui & 0,068 & 0,694 & $\mathbf{0 , 0 1 5}$ \\
\hline & & Non & 0,015 & 0,694 & 0,068 \\
\hline \multirow[t]{6}{*}{ B } & \multirow[t]{2}{*}{ Tailler les arbres } & Oui & 0,068 & 0,678 & $\mathbf{0 , 0 1 5}$ \\
\hline & & Non & 0,015 & 0,678 & 0,068 \\
\hline & \multirow[t]{2}{*}{ Désherber la plantation } & Oui & 0,076 & 0,956 & 0,008 \\
\hline & & Non & 0,008 & 0,956 & 0,076 \\
\hline & \multirow[t]{2}{*}{ Protéger la plantation contre les insectes } & Oui & 0,068 & 0,694 & 0,015 \\
\hline & & Non & 0,015 & 0,694 & 0,068 \\
\hline \multirow[t]{6}{*}{$\mathbf{C}$} & \multirow[t]{2}{*}{ Tailler les arbres } & Oui & 0,068 & 0,678 & $\mathbf{0 , 0 1 5}$ \\
\hline & & Non & 0,015 & 0,678 & 0,068 \\
\hline & \multirow[t]{2}{*}{ Désherber la plantation } & Oui & 0,076 & 0,956 & 0,008 \\
\hline & & Non & 0,008 & 0,956 & 0,076 \\
\hline & \multirow[t]{2}{*}{ Protéger la plantation contre les insectes } & Oui & 0,068 & 0,694 & $\mathbf{0 , 0 1 5}$ \\
\hline & & Non & 0,015 & 0,694 & 0,068 \\
\hline \multirow[t]{6}{*}{ D } & \multirow[t]{2}{*}{ Tailler les arbres } & Oui & 0,061 & 0,607 & $\mathbf{0 , 0 2 3}$ \\
\hline & & Non & 0,023 & 0,607 & 0,061 \\
\hline & \multirow[t]{2}{*}{ Désherber la plantation } & Oui & 0,076 & 0,956 & 0,008 \\
\hline & & Non & 0,008 & 0,956 & 0,076 \\
\hline & \multirow[t]{2}{*}{ Protéger la plantation contre les insectes } & Oui & 0,068 & 0,694 & 0,015 \\
\hline & & Non & 0,015 & 0,694 & 0,068 \\
\hline \multicolumn{5}{|l|}{ Moyenne } & 0,013 \\
\hline
\end{tabular}

Source : l'auteur à partir de l'enquête CCA-iCA 2015 